\title{
INTERFERÊNCIA DE CARURU-DE-MANCHA, MARIA-PRETINHA, PICÃO-PRETO E TIRIRICA EM TOMATEIRO INDUSTRIAL $\left({ }^{\mathbf{1}}\right)$
}

\author{
BRUNA PIRES DA SILVA $\left({ }^{2}\right)$; LEONARDO BIANCO DE CARVALHO $\left(3^{*}\right)$; \\ PEDRO LUIS DA COSTA AGUIAR ALVES $\left({ }^{4}\right)$; MARCELO CLARO DE SOUZA $\left({ }^{5}\right)$; \\ FERNANDO BENINI MAGÁRIO ( $\left.{ }^{2}\right)$
}

\begin{abstract}
RESUMO
O objetivo da presente pesquisa foi estudar o efeito da interferência de caruru-de-mancha (Amaranthus viridis), picão-preto (Bidens pilosa), tiririca (Cyperus rotundus) e Maria-pretinha (Solanum americanum) sobre a altura de plantas, a área foliar, o teor de clorofila, o acúmulo de massa seca e macronutrientes na cultura e a massa fresca de frutos do tomateiro industrial. Os tratamentos foram (a) duas plantas de tomateiro + duas de A. viridis; (b) duas plantas de tomateiro + duas de B. pilosa; (c) duas plantas de tomateiro + duas de C. rotundus; e (d) duas plantas de tomateiro + duas de $S$. americanum. Além disso, manteve-se uma testemunha com duas plantas de tomateiro sem associação com planta daninha. Dentro de cada espécie de planta daninha, o efeito sobre a cultura foi o mesmo para todas as características avaliadas, independentemente da distância utilizada. A. viridis e $S$. americanum foram plantas daninhas com maior capacidade competitiva que $B$. pilosa e $C$. rotundus. O teor de clorofila, a altura e a área foliar do tomateiro não foram afetados pela convivência com as plantas daninhas. B. pilosa e C. rotundus não influenciaram no acúmulo de macronutrientes do tomateiro, enquanto $A$. viridis e $S$. americanum afetaram de maneira diferenciada em função da distância utilizada, sendo A. viridis a espécie mais competitiva. A massa fresca de frutos do tomateiro não foi reduzida pela convivência com as quatro espécies de plantas daninhas.
\end{abstract}

Palavras-chave: tomate, plantas daninhas, competição, crescimento, macronutrientes.

\section{ABSTRACT \\ INTERFERENCE OF SLENDER AMARANTH, AMERICAN BLACK NIGHTSHADE, HAIRY BEGGARTICKS AND PURPLE NUTSEDGE ON PROCESSING TOMATO}

The objective of the present research was to study the effect of interference of slender amaranth (Amaranthus viridis), American black nightshade (Bidens pilosa), purple nutsedge (Cyperus rotundus) and hairy beggarticks (Solanum americanum) on height, leaf area, chlorophyll content, dry mass and macronutrients accumulation and fruits fresh mass of processing tomato. The treatments were: (a) two tomato plants + two ones of A. viridis; (b) two tomato plants + two ones of B. pilosa; (c) two tomato plants + two ones of C. rotundus; e (d) two tomato plants + two ones of $S$. americanum. Moreover, a weed-free check with two tomato plants was maintained. For each weed species, the effect on tomato was the same for all evaluated characteristics, independently to distance used. A. viridis and S. americanum showed higher competitive potential than B. pilosa and C. rotundus. Chlorophyll content, plant height and leaf area of tomato was not affected by weed coexistence. B. pilosa and C. rotundus did not influenced on tomato macronutrients accumulation while A. viridis and S. americanum affected differentially it in function of distance used, and $A$. viridis was the most competitive species. Fresh mass of tomato fruits was not reduced due to coexistence with the four weed species.

Key words: tomato, weeds, interference, growth, macronutrients.

$\left({ }^{1}\right)$ Recebido para publicação em 20 de agosto de 2009 e aceito em 3 de dezembro de 2009.

$\left({ }^{2}\right)$ FCAV/UNESP, Curso de Agronomia. E-mail: brunapiresagro@yahoo.com.br; fmagario@yahoo.com.br.

$\left({ }^{3}\right)$ Doutorando em Agronomia, FCAV/UNESP (Produção Vegetal), Via de acesso Prof. Paulo Donato Castellane, s/n, 14884-900 Jaboticabal (SP). Bolsista CNPq. E-mail: agrolbcarvalho@yahoo.com.br $\left(^{*}\right)$ Autor correspondente.

$\left({ }^{4}\right)$ Departamento de Biologia Aplicada à Agropecuária, FCAV/UNESP, Jaboticabal (SP). E-mail: plalves@fcav.unesp.br.

$\left(^{5}\right)$ Doutorando em Biologia Vegetal, Instituto de Biologia, UNESP, Av. 24A, 13506-900 Rio Claro (SP). E-mail: marcelo_claro@yahoo.com.br 


\section{INTRODUÇÃO}

O tomate (Lycopersicum esculentum L.) é uma das mais importantes hortaliças produzidas no mundo (SANTOS, 2009). O Brasil destaca-se como um dos maiores produtores dessa cultura, considerando a produção destinada à indústria, com área colhida de aproximadamente 55.000 hectares e uma produção de cerca de 3.200 .000 toneladas, atingindo a produtividade de $58.000 \mathrm{~kg} \mathrm{ha}^{-1} \mathrm{em} 2007$ (Agrianual, 2008). Entretanto, o tomateiro está sujeito à interferência negativa de plantas daninhas que competem por nutrientes, água e luz e podem causar sérios prejuízos à cultura, reduzindo assim sua produtividade (NASCENTE et al., 2004; HiLlger et al., 2006).

Dentre as plantas daninhas que ocorrem em áreas hortícolas, caruru-de-mancha (Amaranthus viridis), picão-preto (Bidens pilosa), tiririca (Cyperus rotundus) e Maria-pretinha (Solanum americanum) foram destacadas como espécies de grande importância, causando prejuízos em várias hortaliças, incluindo o tomateiro (ZanAtTA et al., 2006). Sabe-se que as plantas daninhas afetam o crescimento e o desenvolvimento das culturas em função da competição por água, luz e, sobretudo, nutrientes (RADOSEVICH et al., 1997). Contudo, a distância em que a planta daninha esteja estabelecida em relação às plantas da cultura de interesse pode determinar maior ou menor grau de interferência (CASADEI, 2007; MarColini et al., 2009), desde que os recursos do meio estejam limitados. Além disso, o grau de interferência varia em função da espécie de planta daninha que esteja competindo com a cultura pelos recursos limitados do meio (Pitelli, 1985).

A hipótese de trabalho é que quatro espécies de plantas daninhas, mantidas em duas distâncias da linha de plantio do tomateiro, afetarão diferencialmente o crescimento e a nutrição mineral da cultura. Assim, o objetivo foi estudar o efeito da interferência de A. viridis, B. pilosa, C. rotundus e $S$. americanum no tomateiro industrial.

\section{MATERIAL E MÉTODOS}

O experimento foi realizado em caixas de cimento amianto com dimensões de 0,50 × 0,50 × 0,30 $\mathrm{m}$, preenchidas com solo coletado na camada arável de Latossolo Vermelho-Escuro, e mantidas a céu aberto, no período de dezembro de 2008 a abril de 2009. Após o enchimento das caixas, coletou-se uma amostra composta do substrato que foi submetida à análise química. Mediante os resultados da análise de solo, aplicou-se calcário e fez-se adubação mineral de plantio e cobertura, seguindo recomendação de TRANI et al. (1997).
As mudas de tomate híbrido HEINZ 9992 e das plantas daninhas foram formadas em bandejas de poliestireno expandido de 128 células preenchidas com substrato Plantmax HT, a partir de sementes (A. viridis, B. pilosa e S. americanum) ou tubérculos (C. rotundus) coletados em área agrícola. Fungicidas e inseticidas foram aplicados desde o estádio de plântulas até a fase adulta do tomateiro e das plantas daninhas, bem como, realizadas as regas periodicas ao longo do ciclo.

O experimento constou de nove tratamentos, estabelecidos em delineamento inteiramente casualizado, com quatro repetições. O transplante das mudas ocorreu 40 dias após a semeadura das bandejas. Duas mudas de tomateiro foram transplantadas na linha central das caixas, distanciadas de $30 \mathrm{~cm}$, sendo, em seguida, transplantadas as plantas daninhas, de modo a estabelecer os tratamentos: (a) duas plantas de tomateiro + duas de A. viridis; (b) duas plantas de tomateiro + duas de B. pilosa; (c) duas plantas de tomateiro + duas de C. rotundus; e (d) duas plantas de tomateiro + duas de S. americanum. As plantas daninhas foram transplantadas uma de cada lado e perpendicularmente à linha de plantio do tomateiro, na linha central das caixas. Os tratamentos foram aplicados para o espaçamento de 5 e $10 \mathrm{~cm}$ em que as plantas daninhas foram mantidas distanciadas da linha de plantio do tomateiro. Além disso, mantevese uma testemunha com duas plantas de tomateiro sem associação com planta daninha.

Decorridos 120 dias após o transplante, quando $80 \%$ dos frutos de tomate estavam maduros, determinou-se a altura de plantas e o teor relativo de clorofila total do tomateiro. Em seguida, as plantas de tomate foram colhidas e separadas em caule, folhas e frutos, para determinação da área foliar, massa fresca de frutos e, posteriormente, da massa seca total. Concomitantemente, as plantas daninhas também foram colhidas para determinação da massa seca (parte aérea de A. viridis, B. pilosa e S. americanum; e planta inteira de $C$. rotundus). Além disso, determinou-se o acúmulo de macronutrientes em folhas do tomateiro, multiplicando-se a massa seca de folhas pelo teor de nutrientes contido nas folhas.

A altura de plantas foi medida da base da planta até a inserção da última folha, utilizando régua graduada. O teor relativo de clorofila total foi determinado utilizando SPAD 502, fazendo a leitura na terceira folha totalmente expandida. A área foliar foi medida utilizando LI-COR, modelo LI 3000A. A massa seca foi determinada mediante prévia lavagem do material verde, de acordo com SARRUGE e HAAG (1974), e secagem em estufa a 70 C por 96 horas, sendo 
o material seco posteriormente pesado em balança eletrônica de precisão de 0,01 g. Em seguida, o material seco foi moído e encaminhado para a determinação dos teores de nitrogênio total $(\mathrm{N})$ e fósforo (P), segundo SARRUGe e HAAG (1974), potássio $(\mathrm{K})$, cálcio $(\mathrm{Ca})$ e magnésio $(\mathrm{Mg})$, segundo JorGENSEN (1977), e enxofre (S), segundo VITTi (1989). O acúmulo de macronutrientes foi calculado multiplicando-se o acúmulo de matéria seca da folha pelo teor do nutriente. A massa fresca de frutos foi determinada por meio de pesagem em balança de precisão de $0,01 \mathrm{~g}$.

Todos os dados foram submetidos à análise de variância pelo teste $\mathrm{F}$, sendo as médias comparadas pelo teste de Tukey, a 5\% de probabilidade, utilizando-se do software SISVAR 5.1 (FERREIRA, 2007).

\section{RESULTADOS E DISCUSSÃO}

As quatro espécies de plantas daninhas acumularam massa seca diferentemente entre si, porém não foi observada diferença no acúmulo quando comparadas as distâncias de estabelecimento dentro da mesma espécie (Tabela 1 ). A. viridis e $S$. americanum acumularam mais massa seca que $B$. pilosa e C. rotundus, com exceção de $B$. pilosa a $5 \mathrm{~cm}$, independentemente da distância, Além disso, B. pilosa e C. rotundus acumularam quantidade semelhante de massa seca. Tais fatos evidenciam que, no geral, plantas de $A$. viridis e $S$. americanum cresceram mais que $B$. pilosa e $C$. rotundus, podendo assim se notar maior potencial de interferência à cultura.

Tabela 1. Massa seca acumulada por quatro espécies de plantas daninhas mantidas a duas distâncias da linha de plantio do tomateiro

\begin{tabular}{lcc}
\hline Espécies & Distância & Massa seca $\left(^{1}\right)$ \\
\hline \multirow{2}{*}{ SOLAM } & $\mathrm{cm}$ & g planta $^{-1}$ \\
& 5 & $52,0 \pm 9,5 \mathrm{ab}$ \\
BIDPI & 10 & $64,1 \pm 3,0 \mathrm{a}$ \\
& 5 & $27,3 \pm 6,8 \mathrm{bc}$ \\
AMAVI & 10 & $18,7 \pm 1,1 \mathrm{c}$ \\
& 5 & $67,1 \pm 5,7 \mathrm{a}$ \\
CYPRO & 10 & $55,5 \pm 8,8 \mathrm{a}$ \\
& 5 & $5,4 \pm 2,0 \mathrm{c}$ \\
CV $(\%)$ & 10 & $4,0 \pm 0,4 \mathrm{c}$ \\
DMS & - & 31,1 \\
\hline
\end{tabular}

$\left({ }^{1}\right)$ Significativo a $5 \%$ de probabilidade pelo teste $F$; médias \pm o erropadrão seguidas pela mesma letra na coluna não diferem entre si pelo teste de Tukey $(\mathrm{p}<0,05)$. SOLAM - Solanum americanum, BIDPI - Bidens pilosa, AMAVI - Amaranthus viridis e CYPRO - Cyperus rotundus.
O teor relativo de clorofila total, a altura de plantas e a área foliar do tomateiro infestado pelas quatro plantas daninhas não diferiram daqueles da testemunha sem convivência, havendo, no entanto, diferença no acúmulo de massa seca (Tabela 2). A massa seca da cultura submetida à convivência com C. rotundus foi semelhante àquela da testemunha, independentemente da distância, assim como com $B$. pilosa a $10 \mathrm{~cm}$. Considerando ainda a interferência de cada espécie daninha, não foi observada diferença no acúmulo de massa seca do tomateiro em função da distância. Tais fatos evidenciam que cada espécie de planta daninha afeta o crescimento do tomateiro independentemente da distância, salvo as exceções supracitadas.

Corroborando os resultados deste experimento, foi observada redução no acúmulo de massa seca pelo tomateiro em consequência da interferência de Abutilon theophrasti (Ngouajo et al., 2001), capim-arroz (Echinochloa crus-galli) (NorRIs et al., 2001) e S. americanum (HERNANDEZ et al., 2002); a altura de plantas não foi influenciada devido à interferência de $A$. theophrasti. Contudo, foi verificada redução na área foliar do tomateiro mantido em convivência com $E$. crusgalli e S. americanum, não corroborando os resultados deste experimento. Resultados distintos podem ser explicados em função de diferentes condições de infestação e manejo ambiental em que foram desenvolvidos os experimentos.

Em relação à testemunha, B. pilosa e $C$. rotundus não influenciaram os acúmulos de todos os macronutrientes do tomateiro, independentemente da distância, assim como S. americanum a $5 \mathrm{~cm}$ (Tabela 3). Além disso, A. viridis a $5 \mathrm{~cm}$ não afetou os acúmulos de $\mathrm{K}, \mathrm{Ca}, \mathrm{Mg}$ e $\mathrm{S}$, enquanto $\mathrm{S}$. americanum a $10 \mathrm{~cm}$ não afetou os acúmulos de $\mathrm{K}$ e $\mathrm{Mg}$. Considerando ainda a interferência de cada espécie daninha, não foi observada diferença no acúmulo de macronutrientes do tomateiro em função da distância. Tais fatos evidenciam que, no geral, $A$. viridis afetou mais o acúmulo de macronutrientes que $S$. americanum.

Estudando a marcha-de-absorção de macronutrientes em $A$. viridis (BIANCO et al., 2006), B. pilosa (DuARTE et al., 2005) e S. americanum (BIANCo et al., 1997), foi constatado pelos pesquisadores que essas espécies podem ser extremamente agressivas na competição por nutrientes com as plantas cultivadas, com destaque para A. viridis e S. americanum. Além disso, CAtunda et al. (2006) verificaram que $C$. rotundus afetou a absorção de macronutrientes em abacaxizeiro, enquanto $B$. pilosa não interferiu demasiadamente na absorção, demonstrando que em todas as espécies estudadas havia potencial de competição por macronutrientes. 
Tabela 2. Teor de clorofila total (Cl.), altura de plantas (Alt.), área foliar (AF) e massa seca acumulada (MST) pelo tomateiro submetido à convivência com quatro espécies de plantas daninhas, em duas distâncias (Dist.) da linha de plantio

\begin{tabular}{|c|c|c|c|c|c|}
\hline Espécie & Dist. & $\mathrm{Cl}$. & Alt. & $\mathrm{AF}$ & $\operatorname{MST}\left({ }^{1}\right)$ \\
\hline & $\mathrm{cm}-$ & UR- & $\mathrm{cm}$ & $\mathrm{cm}^{2}$ planta $^{-1}$ & g planta ${ }^{-1}$ \\
\hline Testem. & - & $49,5 \pm 2,0$ & $79,4 \pm 2,1$ & $3.356,7 \pm 639,8$ & $109,5 \pm 10,5 \mathrm{a}$ \\
\hline \multirow[t]{2}{*}{ SOLAM } & 5 & $40,5 \pm 4,0$ & $73,6 \pm 5,6$ & $2.356,9 \pm 407,9$ & $64,3 \pm 6,0 \mathrm{bc}$ \\
\hline & 10 & $44,0 \pm 2,6$ & $66,6 \pm 2,7$ & $1.407,5 \pm 336,6$ & $40,5 \pm 6,6 c$ \\
\hline \multirow[t]{2}{*}{ BIDPI } & 5 & $44,2 \pm 0,7$ & $60,6 \pm 10,9$ & $3.798,5 \pm 1.022,3$ & $66,2 \pm 5,2 b c$ \\
\hline & 10 & $42,3 \pm 2,7$ & $76,9 \pm 2,8$ & $4.038,3 \pm 705,6$ & $89,5 \pm 6,5 \mathrm{ab}$ \\
\hline \multirow[t]{2}{*}{ AMAVI } & 5 & $38,4 \pm 2,1$ & $66,6 \pm 6,0$ & $1.854,3 \pm 237,3$ & $43,4 \pm 4,2 \mathrm{c}$ \\
\hline & 10 & $38,4 \pm 1,5$ & $68,2 \pm 6,0$ & $1.480,2 \pm 378,0$ & $30,9 \pm 6,2 c$ \\
\hline \multirow[t]{2}{*}{ CYPRO } & 5 & $45,8 \pm 2,5$ & $73,2 \pm 9,1$ & $3.616,2 \pm 435,7$ & $72,7 \pm 13,7 \mathrm{abc}$ \\
\hline & 10 & $41,9 \pm 2,1$ & $66,5 \pm 9,2$ & $3.148,2 \pm 444,7$ & $90,4 \pm 14,7 \mathrm{ab}$ \\
\hline CV $(\%)$ & - & 12,9 & 19,2 & 40,2 & 26,5 \\
\hline DMS & - & 13,2 & 6,7 & 559,6 & 42,6 \\
\hline
\end{tabular}

$\left({ }^{1}\right)$ Significativo a $5 \%$ de probabilidade pelo teste F; médias \pm o erro padrão seguidas pela mesma letra na coluna não diferem entre si pelo teste de Tukey $(\mathrm{p}<0,05)$. SOLAM - Solanum americanum, BIDPI - Bidens pilosa, AMAVI - Amaranthus viridis e CYPRO - Cyperus rotundus.

É interessante destacar que, embora a massa seca total e o acúmulo de macronutrientes terem sido afetados, principalmente pela convivência com $A$. viridis e $S$. americanum, a massa fresca de frutos, característica de interesse econômico, não foi reduzida devido à convivência com as quatro espécies de plantas daninhas, quando comparada àquela da testemunha (Tabela 4). Contudo, houve diferença apenas entre a cultura que conviveu com $A$. viridis a $10 \mathrm{~cm}$ e C. rotundus a $10 \mathrm{~cm}$. Tal fato pode ser explicado, pois a planta, ao atingir o estádio de florescimento, altera seu órgão-dreno, direcionando a maior parte dos assimilados para a estrutura reprodutiva (TAIZ e Zieger, 2002). Assim, se a competição por água, luz e nutrientes não for intensamente limitante, o desenvolvimento do fruto pode não ser afetado.

Esse resultado não corrobora aqueles obtidos por NgOuajio et al. (2001), Norris et al. (2001), Nascente et al. (2004), Hillger et al. (2006) e HERNANDEZ et al. (2007), entre outros, que relataram intensa redução na produção de frutos do tomateiro em decorrência da interferência imposta por plantas daninhas, que pode atingir mais de $90 \%$ quando a comunidade infestante convive com a cultura durante todo o ciclo agrícola. Deve-se ressaltar que esses autores observaram distintas reduções na produção de frutos, o que pode ser atribuído ao fato de as comunidades infestantes serem compostas por distintas espécies de plantas daninhas, além das condições edafo-climáticas terem sido diferentes.
O fato de as plantas daninhas não terem afetado a massa seca de frutos do tomateiro pode ter ocorrido em função da utilização da densidade de apenas duas plantas daninhas competindo com a cultura em uma área de $0,25 \mathrm{~m}^{2}\left(8\right.$ plantas $\left.\mathrm{m}^{-2}\right)$. A densidade de infestação de plantas daninhas é fator importante na determinação da intensidade de redução da produção do tomateiro (HERNANDEZ et al., 2002), devido ao aumento do número de plantas daninhas promover diminuição na quantidade de radiação fotossinteticamente ativa disponível à cultura (NoRRIS et al., 2001), o que acarreta redução na produção. Portanto, provavelmente, a densidade de infestação foi baixa, por isso não houve redução na massa seca de frutos.

Em decorrência dessa baixa densidade de infestação, as plantas daninhas não puderam expressar seu potencial de agressividade, que advém principalmente de sua plasticidade fenotípica e alta produção de propágulos, fundamentais para colonização de áreas sem cobertura vegetal. Assim, plantas de pequeno porte, como C. rotundus, que acumulam pouca massa individual, expressam seu potencial competitivo apenas em altas densidades. Isso pode ser evidenciado, ainda, pelo fato de $A$. viridis e $S$. americanum terem sido mais competitivas com o tomateiro em relação a C. rotundus e B. pilosa, duas espécies que acumularam menos massa que as duas primeiras, na mesma densidade de plantas. 
Tabela 3. Acúmulo de macronutrientes em folhas de tomateiro submetido à convivência com quatro espécies de plantas daninhas, em duas distâncias da linha de plantio

\begin{tabular}{|c|c|c|c|c|c|c|c|}
\hline Espécie & Dist. & $N\left({ }^{1}\right)$ & $\mathrm{P}\left({ }^{1}\right)$ & $\mathrm{K}\left({ }^{1}\right)$ & $\mathrm{Ca}\left({ }^{1}\right)$ & $\operatorname{Mg}\left({ }^{1}\right)$ & $S\left({ }^{1}\right)$ \\
\hline & & & & $-\mathrm{n}$ & $\operatorname{anta}^{-1}$ & & \\
\hline Testem. & $\mathrm{cm}$ & $797,3 \pm 150,2 \mathrm{a}$ & $98,7 \pm 21,0 \mathrm{a}$ & $938,7 \pm 241,2 \mathrm{a}$ & $752,2 \pm 177,8 \mathrm{a}$ & $225,1 \pm 51,7 \mathrm{ab}$ & $120,6 \pm 24,9 \mathrm{a}$ \\
\hline \multirow[t]{2}{*}{ SOLAM } & 5 & $517,7 \pm 61,3 \mathrm{abc}$ & $57,89 \pm 5,86$ abcd & $683,8 \pm 68,1 \mathrm{ab}$ & $482,6 \pm 45,7 \mathrm{ab}$ & $141,7 \pm 16,1 \mathrm{bc}$ & $80,4 \pm 6,9 a b c$ \\
\hline & 10 & $272,9 \pm 46,8 \mathrm{bc}$ & $29,2 \pm 4,3 \mathrm{~d}$ & $360,1 \pm 59,9 a b$ & $269,8 \pm 42,3 b$ & $80,5 \pm 11,3 \mathrm{bc}$ & $42,4 \pm 6,6 \mathrm{c}$ \\
\hline \multirow[t]{2}{*}{ BIDPI } & 5 & $644,1 \pm 49,7 \mathrm{abc}$ & $69,4 \pm 4,9$ abcd & $815,1 \pm 64,0 \mathrm{ab}$ & $571,9 \pm 51,7 \mathrm{ab}$ & $179,1 \pm 14,7 \mathrm{abc}$ & $101,1 \pm 8,1 \mathrm{abc}$ \\
\hline & 10 & $790,1 \pm 68,2 \mathrm{a}$ & $89,1 \pm 7,6 \mathrm{ab}$ & $947,6 \pm 57,9 \mathrm{a}$ & $679,3 \pm 48,2 \mathrm{ab}$ & $224,3 \pm 16,2 \mathrm{ab}$ & $121,6 \pm 8,0 \mathrm{a}$ \\
\hline \multirow[t]{2}{*}{ AMAVI } & 5 & $330,7 \pm 28,9 \mathrm{bc}$ & $41,1 \pm 3,7 \mathrm{bcd}$ & $397,4 \pm 39,1 \mathrm{ab}$ & $381,2 \pm 32,8 \mathrm{ab}$ & $107,6 \pm 8,5 \mathrm{bc}$ & $61,5 \pm 4,7 \mathrm{abc}$ \\
\hline & 10 & $246,8 \pm 50,8 \mathrm{c}$ & $30,8 \pm 6,0 \mathrm{~cd}$ & $304,5 \pm 58,3 \mathrm{~b}$ & $248,4 \pm 50,7 \mathrm{~b}$ & $71,5 \pm 14,2 \mathrm{c}$ & $45,4 \pm 10,0 \mathrm{bc}$ \\
\hline \multirow[t]{2}{*}{ CYPRO } & 5 & $784,1 \pm 73,8$ a & $83,7 \pm 8,6 \mathrm{abc}$ & $922,9 \pm 127,6 \mathrm{ab}$ & $729,2 \pm 98,3 \mathrm{a}$ & $308,3 \pm 48,5 \mathrm{a}$ & $119,8 \pm 7,3 \mathrm{a}$ \\
\hline & 10 & $681,1 \pm 93,5 \mathrm{ab}$ & $85,5 \pm 12,9 a b$ & $867,8 \pm 147,1 \mathrm{ab}$ & $651,9 \pm 85,3 \mathrm{ab}$ & $190,3 \pm 23,6 a b c$ & $111,0 \pm 17,0 \mathrm{ab}$ \\
\hline $\mathrm{CV}(\%)$ & - & 31,6 & 34,8 & 37,9 & 35,9 & 37,2 & 31,1 \\
\hline DMS & - & 422,7 & 53,9 & 625,9 & 452,6 & 150,2 & 66,2 \\
\hline
\end{tabular}

( $\left.{ }^{1}\right)$ Significativo a $5 \%$ de probabilidade pelo teste $\mathrm{F}$; médias \pm o erro padrão seguidas pela mesma letra na coluna não diferem entre si pelo teste de Tukey $(\mathrm{p}<0,05)$. SOLAM - Solanum americanum, BIDPI - Bidens pilosa, AMAVI - Amaranthus viridis e CYPRO - Cyperus rotundus.

Tabela 4. Massa fresca de frutos de tomate submetido à convivência com quatro espécies de plantas daninhas, em duas distâncias da linha de plantio

\begin{tabular}{lcc}
\hline Espécies & Distância & Massa Fresca $\left(^{1}\right)$ \\
\hline Testemunha & $\mathrm{cm}$ & g planta $^{-1}$ \\
SOLAM & - & $238,2 \pm 43,7 \mathrm{ab}$ \\
& 5 & $112,9 \pm 19,5 \mathrm{ab}$ \\
BIDPI & 10 & $125,9 \pm 20,0 \mathrm{ab}$ \\
& 5 & $126,0 \pm 39,4 \mathrm{ab}$ \\
AMAVI & 10 & $223,2 \pm 44,8 \mathrm{ab}$ \\
CYPRO & 5 & $98,0 \pm 14,7 \mathrm{ab}$ \\
& 10 & $64,7 \pm 7,7 \mathrm{~b}$ \\
CV (\%) & 5 & $111,4 \pm 45,6 \mathrm{ab}$ \\
DMS & 10 & $329,0 \pm 120,8 \mathrm{a}$ \\
\hline
\end{tabular}

$\left.{ }^{1}\right)$ Significativo a $5 \%$ de probabilidade pelo teste F; médias \pm o erro padrão seguidas pela mesma letra na coluna não diferem entre si pelo teste de Tukey $(\mathrm{p}<0,05)$. SOLAM - Solanum americanum, BIDPI - Bidens pilosa, AMAVI - Amaranthus viridis e CYPRO - Cyperus rotundus.

\section{CONCLUSÕES}

1. Dentro de cada espécie de planta daninha, o efeito sobre a cultura é o mesmo para todas as características avaliadas, independentemente da distância utilizada.

2. A. viridis e S. americanum são plantas daninhas com maior capacidade competitiva que $B$. pilosa e C. rotundus, mantidas em convivência com a cultura do tomate industrial.
3. O teor de clorofila, a altura e a área foliar do tomateiro não são afetados pela convivência com as plantas daninhas.

4. B. pilosa e C. rotundus não influenciam no acúmulo de macronutrientes do tomateiro, enquanto $A$. viridis e $S$. americanum afetaram de maneira diferenciada em função da distância utilizada, sendo que $A$. viridis é a espécie mais competitiva.

5. A massa fresca de frutos do tomateiro não é reduzida pela convivência com as quatro espécies de plantas daninhas.

\section{AGRADECIMENTO}

Os autores agradecem ao PIBIC/CNPq pela concessão de Bolsa IC ao primeiro autor.

\section{REFERÊNCIAS}

AGRIANUAL: Anuário da agricultura brasileira. São Paulo: FNP Consultoria, 2008. p.478-482.

BIANCO, S.; CARVALHO, L.B.; BIANCO, M.S.; DUARTE, D.J. Crescimento e acúmulo de macronutrientes por plantas de Amaranthus viridis. In: CONGRESSO BRASILEIRO DA CIÊNCIA DAS PLANTAS DANINHAS, 25., 2006. Anais... Brasília:SBCPD, 2006. p.42.

BIANCO, S.; PITELLI, R.A.; BELLINGIERI, P.A.; PAVANI, M.C.M.D. Crescimento e nutrição mineral de Solanum americanum. In: CONGRESSO BRASILEIRO DA CIÊNCIA DAS PLANTAS DANINHA, 21., 1997. Anais... Caxambu: SBCPD, 1997. p.40. 
CASADEI, E. Efeito da densidade e da distribuição do caruru-gigante sobre quatro cultivares de alface. 2007. $49 \mathrm{f}$. Monografia (Graduação em Agronomia) - Faculdade de Ciências Agrárias e Veterinárias, Universidade Estadual Paulista, Jaboticabal.

CATUNDA, M.G.; FREITAS, S.P.; SILVA, C.M.M.; CARVALHO, A.J.R.C.; SOARES, L.M.S. Interferência de plantas daninhas no acúmulo de nutrientes e no crescimento de plantas de abacaxi. Planta Daninha, v.24, p.199-204, 2006.

DUARTE, D.J.; BIANCO, S.; BIANCO, M.S.; PARREIRA, M.C.; LUVEZUTTI, R.A. Crescimento e nutrição mineral de Bidens pilosa L. In: CONGRESSO DE INICIAÇÃO CIENTÍFICA DA UNESP, 17., 2005. Anais... Jaboticabal: UNESP, 2005. (CD-Rom)

FERREIRA, D.F. Sisvar: sistema de análise de variância para dados balanceados, versão 5.1. Lavras: DEX/ UFLA, 2007. Software estatístico.

HERNANDEZ, D.D.; ALVES, P.L.C.A.; PAVANI, M.C.M.D.; PARREIRA, M.C. Períodos de interferência de maria-pretinha sobre tomateiro industrial. Horticultura Brasileira, v.25, p.199204, 2007.

HERNANDEZ, D.D.; ALVES, P.L.C.A.;SALGADO, T.P. Efeito da densidade e proporção de plantas de tomate industrial e de maria-pretinha em competição. Planta Daninha, v.20, p.229236, 2002.

HILLGER, D.E.; WELLER, S.C.; GIBSON, K.D. Weed management systems in Indiana tomato production. Weed Science, v.54, p.516-520, 2006.

JORGENSEN, S.S. Metodologia utilizada para análises químicas de rotina: guia analítico. Piracicaba: CENA, 1977. 24p.

MARCOLINI, L.W.; ALVES, P.L.C.A.; DIAS, T.C.S.; PARREIRA, M.C. Effect of density and the distance of Brachiaria dcumbens Staff on the initial growth of Coffea arabica. Coffee Science, v. 4, p.11-15, 2009.

NASCENTE, A.S.; PEREIRA, W.; MEDEIROS, M.A. Interferência das plantas daninhas na cultura do tomate para processamento. Horticultura Brasileira, v.22, p.602-606, 2004.

NORRIS, R.F.; ELMORE, C.L.; REJMÁNEK, M. AKEY, W.C. Spatial arrangement, density, and competition between barnyardgrass and tomato: I. Crop growth and yield. Weed Science, v.49, p.61-68. 2001.

NGOUAJIO M.; MCGIFFEN JUNIOR, M.E.; HEMBREE, K.J. Tolerance of tomato cultivars to velvetleaf interference. Weed Science, v.49, p.91-98, 2001.

PITELLI, R.A. Interferência das plantas daninhas nas culturas agrícolas. Informativo Agropecuário, v.11, p.16-27, 1985.

RADOSEVICH, S.; HOLT, J.; GHERSA, C. Weed ecology: implications for management. 2.ed. New York: Wiley, 1997. 588p.
SANTOS, B.M. Drip-applied metam potassium and herbicides as methyl bromide alternatives for Cyperus control in tomato. Crop Protection, v.28, p.68-71, 2009.

SARRUGE, J.R.; HAAG, H.P. Análises químicas em plantas. Piracicaba: ESALQ/USP, 1974.56p.

TAIZ, L.; ZIEGER, E. Plant Physiology. 3.ed. Sunderland: Sinauer, 2002, 690p.

TRANI, P.E.; NAGAI, H.; PASSOS, F.A. Tomate rasteiro (industrial) irrigado. In: van RAIJ, B.; CANTARELLA, H.; QUAGGIO, J.A.; FURLANI, A.M.C. (Eds.). Recomendações de adubação e calagem para o Estado de São Paulo. 2.ed. Campinas: IAC. 1997, p.185. (Boletim Técnico, 100)

VITTI, G.C. Avaliação e interpretação do enxofre no solo e na planta. Jaboticabal: FUNEP, 1989. 37p.

ZANATTA, J.F.; FIGUEIREDO, S.; FONTANA, L.C.; PROCÓPIO, S.O. Interferência de plantas daninhas em culturas olerícolas. Revista FZVA, v.13, p.39-57, 2006. 